\title{
Average Energy of Sulfur-35 Beta Decay
}

\author{
Howard H. Seliger, Wilfrid B. Mann, and Lucy M. Cavallo
}

\begin{abstract}
The rate of energy emission in an approximately 400-millicurie source of sulfur-35 was measured by means of a "radiation-balance" microcalorimeter. This value and the disintegration rate of an accurate dilution of the sample measured by means of $4 \pi \beta$-gas-proportional counting yield a mean energy for sulfur-35 beta decay of $50.4\left({ }_{-1.2}^{+0.7}\right) \mathrm{kev}$. The half-life has been determined to be $87.16 \pm 0.1$ days.
\end{abstract}

\section{Introduction}

The rate of energy emission in approximately $400 \mathrm{mc}$ of sulfur-35 was recently measured by means of the radiation balance $[1,2]^{1}$ for the purpose of determining $W_{\mathrm{air}}$, the average energy expended to produce one ion pair in air by the beta particles emitted by sulfur-35 [3, 4]. A part of this sample of sulfur-35, which was of high specific activity, was subsequently used to prepare radioactivity solution standards of the nuclide. From these standards a number of sources having extremely low solid content were subsequently prepared for $4 \pi \beta$ counting $[5,6,7]$. These low-solids sources were prepared in the same manner as for the determination of $W_{\text {air }}$ at the National Bureau of Standards [4].

The average energy of the sulfur-35 beta decay was derived from the results of disintegration rate measurements by $4 \pi \beta$ counting and the rate of energy emission obtained from the radiation balance.

\section{Radiation-Balance Measurements}

The sample of sulfur-35 consisted of about $400 \mathrm{mc}$ of sodium sulfate sealed in a soft-glass tube $3.3 \mathrm{~mm}$ in external diameter and $35 \mathrm{~mm}$ in length. A dummy source of the same dimensions containing inactive sodium sulfate was also prepared for use in the second cup of the radiation balance.

It so happened that there were available in the laboratory at the time two other radioactive preparations that were emitting energy at about the same rate as the sulfur-35 sample. One of these was a polonium-210 sample that had been used to measure the branching ratio in the decay of polonium-210 [8], and the other was a sample of about 4.8 curies of tritiated water sealed in a quartz tube under a partial pressure of about $2 \mathrm{~cm} \mathrm{Hg}$ of hydrogen. This latter sample was subsequently used to prepare the National Bureau of Standards tritiated-water standards.

For the purpose of checking the internal consistency of the results obtained, it seemed that it would be advantageous to measure the tritium, sulfur-35, and polonium-210 samples together as a triad in the radiation balance in the manner described by Connor and Youden for the comparison of four national radium standards [9].

The results of a great many measurements on the polonium-210 preparation have already been reported [8] and were satisfactorily consistent.

1 Figures in brackets indicate the literature references at the end of this paper.
The 4.8-curie tritium preparation was also measured over a period of about 4 months to insure that no error might arise from energy releases due to the chemical effects of radiation which might be expected to vary with time. Here again the consistency of the results was quite satisfactory. The National Bureau of Standards tritiated-water standards prepared from this sample were also subsequently compared by the method of liquid scintillation counting with the tritium standard samples distributed by Atomic Energy of Canada Limited and with the tritium standard of the University of California's Los Alamos Scientific Laboratory. Agreement was obtained to within 1.3 percent with the Canadian standard, and to within 0.7 percent with the Los Alamos standard.

The results of the microcalorimetric comparison of the tritium, sulfur-35, and polonium-210 preparations are shown in table 1 . From these results "best estimates" for each of the three preparations, incorporating the difference measurements as well as measurements on the individual preparations, were obtained in the same way as has been described for the national radium standards [9]. These best estimates are 155.7 $\mu \mathrm{w}$ for the tritium as of May 10, $1955 ; 119.9 \mu \mathrm{W}$ for the sulfur-35 as of $1430 \mathrm{ES}$ T on May 11,1955 ; and $262.6 \mu \mathrm{w}$ for the polonium-210 as of 1315 EST on May 11, 1955.

After measurement, the sulfur-35 preparation was put into solution by breaking the soft-glass container by the simple expedient of heating one end of it in an electrically heated coil and then plunging it, while hot, into cold distilled water.

TABLE 1. Rate, and differences in the rate, of energy emission from preparations of tritium, sulfur-35, and polonium-210

\begin{tabular}{|c|c|l|r|}
\hline \hline 1955 & Time (EST) & Source & $\begin{array}{c}\text { Energy } \\
\text { emission } \\
(\mu \mathrm{W})\end{array}$ \\
\hline May 10 & 1330 & $\mathrm{Po}^{210}$ & \\
May, 10 & 1630 & $\mathrm{~S}^{35}-\mathrm{H}^{3}$ & 264.6 \\
May 10 & 1730 & $\mathrm{H}^{3}$ & 35.1 \\
Mayi11 & 1130 & $\mathrm{Po}^{210}-\mathrm{H}^{3}$ & 154.8 \\
May 11 & 1315 & $\mathrm{Po}^{210} \mathrm{~S}^{35}$ & 106.5 \\
May 11 & 1430 & $\mathrm{~S}^{35}$ & 142.8 \\
& & & 120.0 \\
\hline
\end{tabular}

\section{3. $4 \pi \beta$-Gas-Proportional Counting}

The low-energy beta-ray spectrum of sulfur-35 $\left(E_{\beta} \max =167 \mathrm{kev}\right)$ would normally preclude the determination of the disintegration rate of this nuclide by the $4 \pi \beta$-gas-proportional-counting technique. A self-absorption effect, which could be as 
high as 5 to 10 percent, was observed even for cobalt$60\left(E_{\beta \max }=310 \mathrm{kev}\right)$ [6]. However, the cobalt was in the chemical form of $\mathrm{CoCl}_{2}$, which tends to form appreciably large crystals, whereas the sulfur was incorporated into an $\mathrm{SO}_{4}^{--}$ion. With the active material as a part of a negative ion and in a different environment $\left(\mathrm{H}^{+}\right.$and $\mathrm{SO}_{4}^{-}$ions), it was felt that it might be possible to go to very low-solids concentrations where even agglomerization might be difficult, without the danger of adsorption on the glassware, which usually occurs with positive ions. Because of the high specific activity of the original source it was possible to obtain reasonably high activity $4 \pi \beta$-counter sources even after dilution to low-solids concentration. The mass of sodium sulfate originally measured in the microcalorimeter was approximately $100 \mathrm{mg}$. The final dilution contained only $2.5 \mu \mathrm{g}$ of solids per milliliter. Usually only $0.02 \mathrm{ml}$ was delivered to a $4 \pi \beta$-counter sourcemount, so that the final source contained $0.05 \mu \mathrm{g}$. (The corresponding figure for the cobalt-60 sources prepared from $\mathrm{CoCl}_{2}$ was $1.5 \mu \mathrm{g}$.) Stepwise dilutions were made of the original master solution, using conductivity water and $0.5-N$ redistilled hydrochloric acid with no additional sulfate carrier. If there were any adsorption at this very low concentration of sulfate ions, it was felt that the effect might be magnified in the absence of the strongly acid, $0.5-N$, hydrochloric-acid solution. Therefore, parallel dilutions were made using only conductivity water. Over a period of several months, sources prepared from both solutions gave the same counting rates in the $4 \pi \beta$-counter. This appeared to be strong evidence against the presence of appreciable adsorption effects.

Over extended periods of time, water will etch the surface of a Pyrex glass container. This etching process releases extremely minute silica flakes into the solution, and the additional solid material could increase the self-absorption in a $4 \pi \beta$-counter source. Because the experiments on source preparation extended over a period of a vear, it was considered advisable to look for this effect. In February 1956, some 9 months subsequent to the preparation of the original dilutions in glass flasks, a millicurie of high-specific-activity carrier-free sulfur-35 as $\mathrm{H}_{2} \mathrm{SO}_{4}$ in $0.1-N$ hydrochloric acid was obtained from the Oak Ridge National Laboratory. This material was diluted sufficiently with conductivity water and stored in a quartz flask in which there should be much less etching with time than in the regular Pyrex glass flasks. The ratio of disintegrations per second per milliliter of the new "quartz-flask" solution to the disintegrations per second per milliliter of the older "glass-flask" solutions was obtained to a high degree of precision by the technique of formamide counting [7, 10]. By use of this saturationthickness method the solutions could be compared independently of any minute differences in solids content owing to the possible presence of silica in the glass-flask solutions. $4 \pi \beta$-counter sources were then prepared under identical conditions from these solutions, and the counting rates observed were in the same ratio as the formamide counting rates within the precision of the measurements. This result provided substantial evidence that the effect of silica on the self-absorption was small, if present at all.

Aliquots of solution were delivered to collodion or Formvar-polystyrene laminated films ranging from $2.5 \mu \mathrm{g} / \mathrm{cm}^{2}$ to $10 \mu \mathrm{g} / \mathrm{cm}^{2}$. Six different methods of precipitating the source material were tried. These were, in order of their effectiveness in reducing source self absorption: (a) evaporation in air of the entire aliquot, (b) deposition of the aliquot in approximately 20 droplets covering almost the same area as (a) and evaporation in air, (c) evaporation in air of the aliquot, redissolving in water and re-evaporating in air, (d) evaporation in air, heating under an infrared lamp, redissolving in water plus the addition of a droplet of ammonium hydroxide, (e) evaporation in air, redissolving in water and re-evaporating in a saturated ammonia atmosphere, and finally, (f) evaporation in air without heat, heating under an infrared lamp, redissolving in water, and then drying in a saturated ammonia atmosphere. The tremendous differences in counting rates observed in the $4 \pi \beta$ counter for sources prepared by these different methods are apparent in table 2 , in which the averages of observed counting rates are given for the various methods. It should be emphasized that all sources contained identical amounts of activity.

TABLE 2. Range of values obtained for various source-preparation methods

\begin{tabular}{|c|c|c|c|}
\hline Method & $\begin{array}{c}\text { Number of } \\
\text { sources }\end{array}$ & $\begin{array}{c}\text { Average } \\
\text { value (in } \\
\text { counts per } \\
\text { second) }\end{array}$ & $\begin{array}{c}\text { Individual } \\
\text { standard } \\
\text { deviation } \\
\text { (in counts } \\
\text { per second) }\end{array}$ \\
\cline { 1 - 2 } & & & \\
(a) & 11 & $11.50 \times 10^{2}$ & \pm 0.76 \\
(b) & 6 & 12.16 & \pm 1.10 \\
(c) & 23 & 12.62 & \pm 0.70 \\
(d) & 3 & 12.90 & \pm 0.25 \\
(e) & 58 & 13.08 & \pm 0.75 \\
(f) & 20 & 13.81 & \pm 0.47 \\
\hline
\end{tabular}

Method (f), giving the optimum results, was also used to prepare sources for the measurement of $W_{\text {air }}$ [4]. It was not possible to investigate the structure of method (f) sources as thoroughly as was done for iodine-131 sources, i. e., by shadow electron micrography, as described in reference [7]. Because of the high normality of the solutions, sources prepared for electron micrography were not too stable and usually corroded the stainless-steel supporting grids of the specimen holder. However, it was possible to examine briefly some portions of several sources. These showed one of two forms, either an extremely fine precipitate of particles of the order of $100 \mathrm{~A}$ in diameter or a structure similar to a flat "two-dimensional" crystal of thickness approximately $100 \mathrm{~A}$. In any case it appeared that, by pure empiricism, a method that gave a minimum of self-absorption had been found for depositing the sulfur-35. It is not clear why this method is so much more effective than the others. There is perhaps some physical action on the substrate sa 
well as on the solution caused by the heating, redissolving, etc.

The small but significant spread of values obtained even when sources were prepared by method (f) is an indication that there still remains a small-selfabsorption effect. Based on the observations in the electron microscope and on subsequent measurements of the absorption of sulfur-35 beta rays in thin films, a self-absorption correction of 1 percent was used in calculating the final result. Due to the uncertainty of particle size in the electron microscope this correction could vary over the range $\left( \pm_{0}^{2}\right)$ percent.

Corrections for mounting-film absorption were made by means of the sandwich technique described in reference [6]. Owing to the low energy of the sulfur-35 the aluminum leaf normally used for the sandwich measurements introduced at least a 5-percent film-absorption correction factor. In order, therefore, to check the validity of the previous derivation over this larger range of mounting-film absorption, experiments were performed with much thinner conducting films as well. Observed counting rates as functions of film thickness were plotted for up to five layers of thin films of collodion made conducting by vacuum evaporation of extremely small quantities of aluminum. In these cases absorption amounted to only 1 percent per film. Straight-line extrapolation of this type of transmission curve to zero film thickness gave the same results as the original aluminum-leaf sandwich measurements.

Because the measurements of disintegration rate extended over a long interval, it was of considerable importance to obtain an accurate value for the half-life of sulfur-35. A least-squares analysis of disintegration-rate values obtained in the $4 \pi \beta$ counter with the same sources over a period of 5 months gave a value of $87.16 \pm 0.1$ days. This value was used to correct all data.

As a final check on the accuracy of the $4 \pi \beta$-gasproportional-counter standardization, the solution that had been standardized was compared with a solution standard received from Atomic Energy of Canada Limited, Chalk River, Ontario, the latter having been sent to the National Bureau of Standards as part of a joint international program of intercomparison of radioactivity standards. The sample from Canada was too weak to be measured with high precision by means of the formamide-

TABLE 3. Intercomparison of $A E C L \mathrm{~S}^{35}$ solution standards a

\begin{tabular}{|c|c|c|c|c|c|}
\hline United & \multicolumn{3}{|c|}{ Canada } & \multicolumn{2}{|c|}{ England } \\
\hline $\begin{array}{l}\text { NBS } \\
4 \pi \beta- \\
\text { prop. } \\
\text { counter }\end{array}$ & $\begin{array}{l}\text { A ECL } \\
4 \pi \beta- \\
\text { prop. } \\
\text { counter }\end{array}$ & $\begin{array}{l}\text { A ECL } \\
\text { internal } \\
\text { gas } \\
\text { counter }\end{array}$ & $\begin{array}{l}\text { MeGill U. } \\
4 \pi \beta- \\
\text { prop. } \\
\text { counter }\end{array}$ & $\begin{array}{l}\mathrm{RCH} \text { b } \\
4 \pi-\mathrm{GM} \\
\text { counter }\end{array}$ & $\begin{array}{l}\text { A ERE c } \\
4 \pi-G M \\
\text { counter }\end{array}$ \\
\hline 1. $37_{5}$ & 1. 26 & 1. 36 & d 1.49 & 1. 36 & 1. 32 \\
\hline
\end{tabular}

a Results are given in microcuries per milliliter as of April 6, 1955.

b Royal Cancer Hospital.

A tomic Energy Research Establishment, Harwell

d The apparent disagreement shown here is probably due to solution differences rather than differences in counting techniques, based on previous agreement with other nuclides. counting technique. A portion of the NBS standard solution was therefore diluted with sufficient carrier to be equivalent in solids concentration and in the same range of activity as the Canadian sample. Sources from each standard were prepared on palladium-faced silver disks, and activity ratios were obtained in (a) the top half of the $4 \pi \beta$ counter and (b) the NBS $2 \pi \beta$-ionization chamber. Both of these measurements gave the same ratio within 0.2 percent.

Table 3 summarizes the results obtained for similar samples distributed by AECL to other standardizing laboratories as well as the NBS results as deduced from the above precise comparison.

\section{Results and Discussion}

A rate of energy emission of $119.9 \mu \mathrm{w}$ corresponds to $748.4 \times 10^{12} \mathrm{ev} / \mathrm{sec}$. The total disintegration rate of the original sodium sulfate was $401.5 \mathrm{mc}$. The mean energy per disintegration is therefore $50.4 \mathrm{kev}$. Table 4 gives a summary of the corrections and uncertainties associated with the measurements. The uncertainty of the experimentally determined mean energy is $\left({ }_{-1.2}^{+0.7}\right) \mathrm{kev}$.

This experimentally determined value agrees quite well with the value of $49.4 \mathrm{kev}$ calculated by Shimanskaia [12] from the Fermi distribution. Shimanskaia also reported an experimental value of $52 \pm 3$ kev, using a defined solid-angle Geiger counter for the absolute-activity measurements.

Recently, Loevinger [13] has calculated a value of $48.8 \mathrm{kev}$ for the mean energy of sulfur-35 beta decay, with an uncertainty of \pm 0.75 percent. Jesse and Sadaukis [14] have quoted a value, determined by calculation, of $48.7 \pm 0.25 \mathrm{kev}$.

TABLE 4. Sources of error in measurement of $\bar{E}$

\begin{tabular}{|c|c|}
\hline Source of error & Uncertainty \\
\hline $\begin{array}{l}\text { Source preparation and } 4 \pi \text { counting } \\
\text { Source self-absorption correction }\end{array}$ & $\begin{array}{l}\% \\
\pm 0.51 \\
(+0)\end{array}$ \\
\hline $\begin{array}{l}\text { Half-life correction } \\
\text { Film-absorption correction } \\
\text { Radiation-balance measurement } \\
\text { Dilution of } 400 \text {-me sample }\end{array}$ & $\begin{array}{l} \pm 0.33 \\
\pm 1 \\
\pm 0.3 \\
\pm .3\end{array}$ \\
\hline Total uncertainty & $\left(\begin{array}{l}+1.3 \\
-2.4\end{array}\right)$ \\
\hline
\end{tabular}

\section{References}

[1] W. B. Mann, J. Research NBS 52, 177 (1954) RP2486.

2] W. B. Mann, J. Research NBS 53, 277 (1954) RP2545.

[3] W. Gross, C. Wingate, and G. Failla, Radiology 66, 101 (1956).

[4] Z. Bay, W. B. Mann, H. H. Seliger, and H. O. Wyckoff, Radiation Research $\boldsymbol{\gamma}, 558$ (1957).

[5] H. H. Seliger and L. Cavallo, J. Research NBS 4\%, 41 (1951) RP2226.

[6] W. B. Mann and H. H. Seliger, J. Research NBS 50, 197 (1953) RP2409.

[7] H. H. Seliger and A. Schwebel, Nucleonics 12:7, 54 (1954). 
[8] R. W. Hayward, D. D. Hoppes, and W. B. Mann, J. Research NBS 54, 47 (1955) RP2564.

[9] W. S. Connor and W. J. Youden, J. Research NBS 53, 273 (1954) RP2544.

[10] A. Schwebel, H. S. Isbell, and J. D. Moyer, J. Research NBS 53, 221 (1954) RP2537.

[11] Excerpted from minutes of National Research Council Subcommittee On Beta and Gamma Ray Measurements and Standards, Feb. 10, 1956.
[12] N. S. Shimanskaia, Soviet Phys. JETP 4:3, 355 (1957)

[13] R. Loevinger, Phys. in Med. Biol. 1, 330 (1957).

[14] W. P. Jesse and J. Sadaukis, Phys. Rev., 107, 766 (1957).

Washington, November 13, 1957. 